\title{
Drosophila Hsp90 Gene Can be Upregulated by Ecdysone
}

\author{
Gang Wang \\ Department of Genetics, Weifang Medical University, Weifang, 261053, China \\ Corresponding author, gangwt@126.com
}

Keywords: Drosophila; Ecdysone, hsp90 gene; RNAi; Ecdysone receptor

\begin{abstract}
The Drosophila hsp90 gene can response to responses to temperature stress and all kind of infection. However, the mechanism of how the hsp90 gene is mediated is still unclear. In the present study, we proved that hsp90 gene abundantly expressed during molting and metamorphosis stage. 20-hydroxyecdysone (20E) treatment can induce hsp90 gene expression, the knockdown of USP1 and EcR-A led to a reduced transcript level of hsp90 gene in Drosophila S2 cells. Those results certified that the Drosophila hsp90 gene involved in the ecdysone signaling transduction pathway and act downstream of the ecdysone receptor.
\end{abstract}

\section{Introduction}

Heat shock proteins (hsp) is a molecular chaperones can be induced by heated, all kinds of infection and inflammation [1]. Heat shock protein hsp90 can combine with ligand molecules and mediate signal transduction, cell proliferation, gene regulation and other physiological function. The hsp90 ligand molecules include a lot of transcription factors and other Functional proteins, such as p23 and FK506 [2-4]. human hsp90 protein can combine serine/threonine kinase Akt to block it dephosphorylation and mediate Akt Biological activity [5].

Hsp90 including 3 conserved domains: the N-Terminal domain (NT), the intermediate domain (MD) and a C-Terminal (CT). The NT domain combine with ATP, the hsp90 conformation changed convenient to bind ligand molecular. The MD domain include a ATP enzyme Dependent arginine acid-catalyzed ring, which can combine with the ligand molecules. Hsp90 usually functions as a Dimer, the CT domain responsible for the Hsp90 Dimer formation [6]. Hsp90 may bind with a variety of proteins through the NT, MD, NT and MD domain, or the entire surface [7].

Drosophila hsp90 can combine with the transcription factors Sim which belong to bHLH family to regulate some gene expression [8]. The mechanism of mediating hsp90 protein binding with different ligand molecules is very complex. In this study, using real-time PCR and RNAi method, we proved that the ecdysone signal pathway can mediate Drosophila hsp90 gene expression through it receptors EcR and USP.

\section{Materials and Methods}

Insect Maintenance. The Drosophila were feed used the artificial food containing agar, antiseptic reagents, glucose, cornmeal andyeast, under the 12ligh: $12 \mathrm{~h}$ dark conditions at $25 \pm 2^{\circ} \mathrm{C}$.

Quantitative real-time PCR . The total RNA of the S2 cells and the Drosophila body was isolated according to the manufacturer's instructions. With total RNA as a template, we for synthesis the first strand cDNA. The utensils used for synthesis the first strand cDNA are get rid of RNase and 
sterilization. Take $5 \mu \mathrm{g}$ RNA and pimers each $1 \mu \mathrm{lmixed}$ water bath at $70{ }^{\circ} \mathrm{C}$ for $5 \mathrm{~min}$, immediately ice bath for $5 \mathrm{~min}$, instantaneous centrifugal, and then add The following reagents in order: 5xbuffer, $0.625 \mu 1$ RNase inhibitors, $1.25 \mu 110 \mathrm{mM}$ dNTP, $1 \mu 1$ M-MLV reverse transcription enzyme, DEPC water to $25 \mu \mathrm{L}, 42{ }^{\circ} \mathrm{C}$ incubation for $1 \mathrm{~h}$, then the first strand cDNA was synthesized.

Based on the Drosophila hsp90 gene sequence, the primers were designed for the PCR, hspF: 5'-ccacgaatgctatactcctg-3', and hspR: 5'-ctcctcagtcatgtcctcta-3'. The act5C gene primers was designed as internal standardization, act5CF: 5'-ctggcttcgctgtccacctt-3' and act5CR 5'-gcactttcgctgct gcttcc-3'. the real-time PCR was programmed two steps: the first step, one cycle at $94{ }^{\circ} \mathrm{C}$ for $3 \mathrm{~min}$; the second step, 32 cycles at $94^{\circ} \mathrm{C}$ for $15 \mathrm{~s}, 62{ }^{\circ} \mathrm{C}$ for $15 \mathrm{~s}$. The comparative CT method was used to analyze the results.

Cell Culture and Hormonal Regulation of hsp90 gene. The Drosophila S2 cells were cultured according to the instructions. The 20E (Sigma) which can imitate ecdysone physiological function was added to the cells and the final concentration was $1 \mu \mathrm{M}$. The negative control cells use equal volume of DMSO. After 20E was added, the S2 cells were cultured for $1 \mathrm{~h}, 3 \mathrm{~h}, 6 \mathrm{~h}$ and $12 \mathrm{~h}$ respectively, then isolated total RNA reverse transcript to the first strand cDNA, use real-time PCR investigate the hsp90 gene expression profile. The results from three independent experiments were analyzed and construct a graph.

RNAi in S2 Cell Line. In order to investigate how ecdysone mediate hsp90 gene expression, the dsRNAs of ecdysone receptor EcR-A and USP1 were designed using the RNAi kit (Ambion, Austin). All the primers for synthesize dsRNAs have a T7 binding sequence (5'-gcgt aatacgactcactatagg-3') on the 5' end, which is promote for transcription. The EcR-A dsRNA primers, EcRiF: 5'-T7-aacaacaattag ctgcctct-3', EcRiR: 5'-T7-gtccttcttgctcttcttc-3'; the USP1 dsRNA primers, USP1iF: 5'-T7-caacgg ttctgatgacttca-3' USP1iR: 5'-T7-gtaatgcggaagaggaaca g-3'. The green fluorescent protein (GFP) was designed as negative control; the primers for GFP was GFPiF: 5'-T7-tggtcccaattctcgtggaac-3' GFPiR: 5'-T7-cttgaagttgaccttgatgcc-3'. The dsRNA quantity and quality was detected by $260 \mathrm{~nm}$ spectrophotometryt and $1.2 \%$ agarose electrophoresis.

The S2 cells are continue cultured in six-well plates, when S2 cells growing to 30-50 percent density, the cell transfection was done. The old medium was discarded and replaced with new 2 $\mathrm{mL}$ of serum-free Grace medium, incubation for $1 \mathrm{~h}$ in incubator. $5 \mu \mathrm{g}$ hsp90 dsRNA and $5 \mu \mathrm{l}$ of liposome lipofectamine 2000 diluted in $250 \mu \mathrm{l}$ of serum-free Grace mix gently and placed 20 min at room temperature. Drop this mixture into the culture medium, rocking several times, then plate the culture medium at $27{ }^{\circ} \mathrm{C}$ for $6 \mathrm{~h}$ in the incubator. Transfection of dsRNA $10 \mathrm{~h}$, add the 20E and continue culture the treated cells for $6 \mathrm{~h}$, isolate total RNA and do real-time PCR analysis.

\section{Results}

Hsp90 Gene Expression Increased During Molting and Metamorphosis. to investigate hsp90 gene expression profile during the larval stage, we extracted the total RNA of the third instar larvae at $0 \mathrm{~h}, 12 \mathrm{~h}, 24 \mathrm{~h}, 48 \mathrm{~h}$ and $60 \mathrm{~h}$. Real-time PCR analysis results showed that hsp90 mRNA level during the third instar larvae has two peaks respectively at $0 \mathrm{~h}$ and $60 \mathrm{~h}$, when the larvae body undergoing molting and metamorphosis (Fig. 1). 


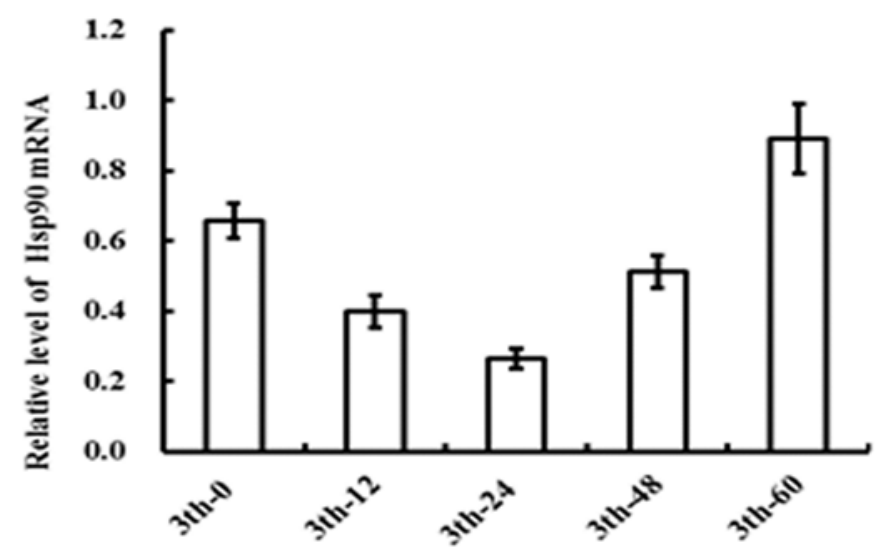

Figure. 1 Real-time PCR analysis of the $h s p 90$ gene expression during the third instar larval from 0 to $60 \mathrm{~h} ;$ act $5 c$ was set as the internal control. Error bars indicate the standard deviation from three independent experiments.

20E Upregulated the Expression of Pvr in S2 Cells. As we all known, during the molting and metamorphosis, Drosophila body ecdysone content increased. In order to detect whether ecdysone can regulate hsp90 gene, we used 20E (Sigma) treat the Drosophila S2 cells for $1 \mathrm{~h}, 3 \mathrm{~h}, 6 \mathrm{~h}, 12 \mathrm{~h}$ respectively. Then extract total RNA and do real-time PCR to analyze the hsp90 gene expression profile. The results showed that the hsp90 gene can be unregulated after the cell was treated $3 \mathrm{~h}$ until $12 \mathrm{~h}$ (Fig. 2). Those results certificated that hsp90 gene can be induced by ecdysone in Drosophila.

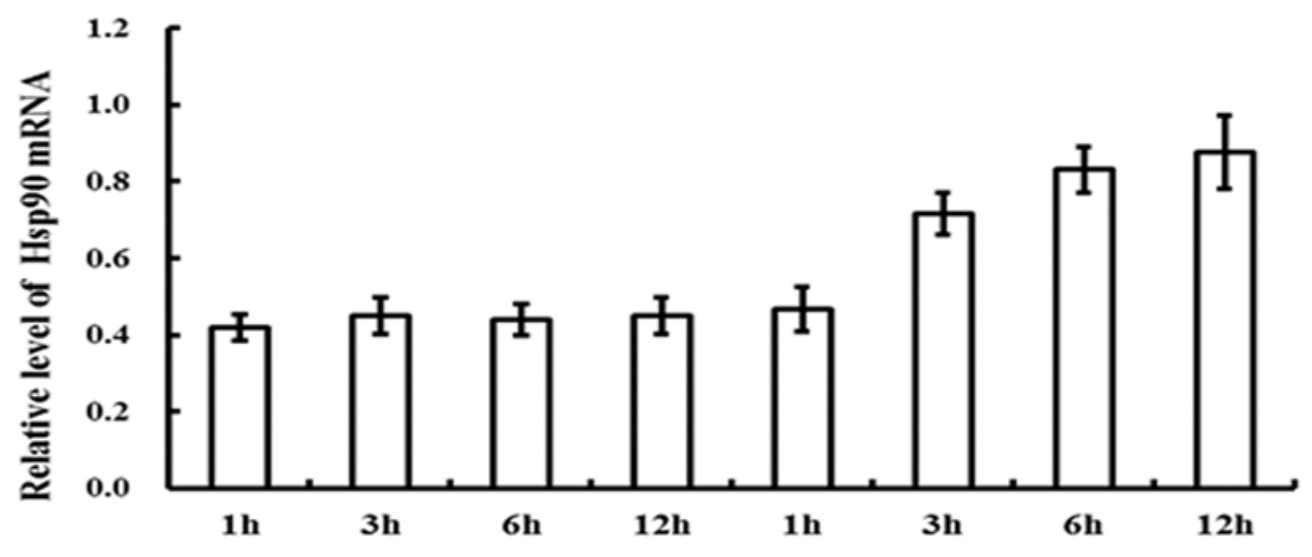

Figure 2. Real-time PCR analysis of the $h s p 90$ gene expression profile $\mathrm{m}$ after the $\mathrm{S} 2$ cell was treated by $20 \mathrm{E}$ at $1,3,6$, and $12 \mathrm{~h}$. The negative control was treated equal DMSO. Error bars indicate the standard deviation of three independent experiments.

Silence of EcR-A and USP1 Blocking the Expression of Pvr. EcR and USP1 are the ecdysone receptor, in order to investigate whether EcR and USP affect the hsp90 gene expression, the EcR isoform EcR-A and USP isoform USP1 were silenced using RNAi method in S2 cells. The real-time PCR results indicated that after the silence of EcR-A and USP1, the 20 E cannot induced the hsp90 expression compared to the negative control which transfected with dsGFP. Those results proved that the hsp90 gene can be mediated by the ecdysone signal transduction pathway and it acts downstream of the EcR and USP (Fig. 3). 


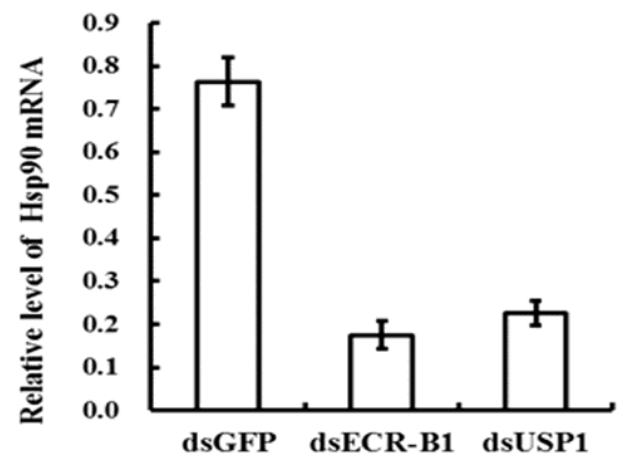

Figure 3. Real-time PCR analyze the $h s p 90$ gene expression from $\mathrm{S} 2$ cells after $E c R-A$ and $U S P I$ were silenced. Error bars indicate the standard deviation from three independent experiments.

\section{Discussion}

In the present study, we investigated the hsp90 gene expression profile using the quantitative real-time PCR and RNAi methods. The results showed that the hsp90 gene mRNA level increased when insect undergoing molting and metamorphosis, and ecdysone can up regulate hsp90 gene expression. The silence of EcR-A and USP1 by RNAi method can block the hsp90 gene expression. Those results proved that Drosophila hsp90 gene involved in the ecdysone signal pathway and it act downstream the ecdysone receptor EcR and USP.

During the holometabolous insects underdoing molting and metamorphosis, the larvae body lose the old integument and produce a new one. During this stage, the insect body exposed to microorganisms, become dangerously and vulnerable. Some immunity genes will be unregulated by ecdysone to protect the insect $[9,10]$. The Insect hsp90 gene can also responses to temperature stress and all kind of infection [1]. So we can propose that ecdysone induce hsp90 gene expression to combat microorganisms during insect molting and metamorphosis stage. However, the detill mechanism need further investigation.

Hsp90 include 3 conserved domains, NT, MD and CT, those three domain can combine other protein and mediate some physiological function such as signal transduction, cell proliferation, gene regulation [11], search the protein can combine with hsp90 will help us understand the mechanism of how hsp90 response to infection.

\section{Acknowledgements}

This research was supported by the Scientific Funds for Outstanding Young Scientists of Shandong Province Award [BS2013SW030], the Project of the Shandong Province Higher Educational Science and Technology Program (J12LK01) and the National Natural Science Foundation of China (81471048).

\section{References}

[1] A. De Maio, "Heat shock proteins: facts, thoughts, and dreams," Shock, vol. 11, pp. 1-12, Jan 1999.

[2] K. N. Jha, A. R. Coleman, L. Wong, A. M. Salicioni, E. Howcroft, and G. R. Johnson, "Heat shock protein 90 functions to stabilize and activate the testis-specific serine/threonine kinases, a family of kinases essential for male fertility," J Biol Chem, vol. 288, pp. 16308-20, Jun 72013. 
[3] M. Taipale, I. Krykbaeva, M. Koeva, C. Kayatekin, K. D. Westover, G. I. Karras, et al., "Quantitative analysis of HSP90-client interactions reveals principles of substrate recognition," Cell, vol. 150, pp. 987-1001, Aug 312012.

[4] A. Zuehlke and J. L. Johnson, "Hsp90 and co-chaperones twist the functions of diverse client proteins," Biopolymers, vol. 93, pp. 211-7, Mar 2010.

[5] S. Sato, N. Fujita, and T. Tsuruo, "Modulation of Akt kinase activity by binding to Hsp90," Proc Natl Acad Sci U S A, vol. 97, pp. 10832-7, Sep 262000.

[6] S. E. Jackson, "Hsp90: structure and function," Top Curr Chem, vol. 328, pp. 155-240, 2013.

[7] G. Chiosis, C. A. Dickey, and J. L. Johnson, "A global view of Hsp90 functions," Nat Struct Mol Biol, vol. 20, pp. 1-4, Jan 2013.

[8] J. McGuire, P. Coumailleau, M. L. Whitelaw, J. A. Gustafsson, and L. Poellinger, "The basic helix-loop-helix/PAS factor Sim is associated with hsp90. Implications for regulation by interaction with partner factors," J Biol Chem, vol. 270, pp. 31353-7, Dec 291995.

[9] J. A. Candido-Silva, G. M. Zanarotti, A. P. Gallina, and J. C. de Almeida, "Developmental regulation of BhSGAMP-1, a gene encoding an antimicrobial peptide in the salivary glands of Bradysia hygida (Diptera, Sciaridae)," Genesis, vol. 45, pp. 630-8, Oct 2007.

[10] G. Wang, P. C. Liu, J. X. Wang, and X. F. Zhao, "A BTB domain-containing gene is upregulated by immune challenge," Arch Insect Biochem Physiol, vol. 77, pp. 58-71, Jun 2011.

[11] F. Burrows, H. Zhang, and A. Kamal, "Hsp90 activation and cell cycle regulation," Cell Cycle, vol. 3, pp. 1530-6, Dec 2004. 